\title{
ESTUDO DOS MÉTODOS DE TRABALHO COM O AUXÍLIO DO MAPEAMENTO DE PROCESSOS PARA O AUMENTO DA PRODUTIVIDADE EM UMA INDÚSTRIA ALIMENTÍCIA
}

\author{
Thalyta Fernanda Gomes de Sousa (UFPE) thalyta5@hotmail.com \\ Manoel Lopes de Andrade Neto (UFPE) manoel.lopes.netto@gmail.com \\ Marcos Antonio Sales Guimarães (UFPE) marcos_asg2_@outlook.com \\ Marcele Elisa Fontana (UFPE) marcele.elisa@ufpe.pe
}

\section{Resumo}

O aumento da necessidade de implantar métodos eficientes de produção por parte das organizações vem crescendo em larga escala devido ao surgimento de novos mercados. Esta exigência impulsiona o desenvolvimento das organizações com a finalidade de melhorar os processos produtivos, seja na redução dos custos produtivos e de tempos de produção, aumento da qualidade do produto, garantia da satisfação dos clientes, assertividade nas tomadas de decisão, entre outros. Com foco no aumento da produtividade, esta pesquisa teve por objetivo analisar os métodos de produção executados por operadores e identificar problemas e soluções para a melhoria do processo de fabricação do biscoito com o auxílio do mapeamento de processos. Como resultado obteve-se a diminuição do tempo padrão total de produção da primeira fase do biscoito Cream Cracker por meio do estudo de tempos e movimentos e pela rápida identificação das melhorias com o auxílio do Software Bizagi Process Modeler (BPM) para a elaboração do mapeamento do processo.

Palavras-Chave: Estudo de Tempos e Movimentos; Redução de Desperdício de Tempo; BPM; Indústria Alimentícia.

\section{Introdução}

A competição globalizada tem ocasionado diversas mudanças no cenário industrial, decorrente, especialmente, do crescente número de produtos e novas marcas que surgem frequentemente no mercado. Com isso, surge a urgência a alguns aspectos tais como maior flexibilidade nos processos, agilidade na tomada de decisão e obtenção do menor custo possível de fabricação. Para tanto, as empresas são desafiadas, constantemente, no processo de implementação de melhorias. 
A melhoria nos processos produtivos pode ser obtida a partir de análises e observações dos métodos de produção com o foco na redução das ações operacionais desnecessárias e aumento na eficiência produtiva. $\mathrm{O}$ estudo dos métodos consiste em analisar as atividades produtivas e garantir que os processos vão ser executados de maneira correta. Contudo, o fato da empresa, objeto de estudo deste trabalho, apresentar métodos de trabalho não padronizados, impossibilita a visualização nítida das atividades desempenhadas pelos operadores, além de dificultar o processo de implementação de melhorias, ocasionando, assim, a insatisfação dos colaboradores e os desperdícios de tempo e materiais na organização. Em relação aos produtos fabricados na empresa analisada foi possível notar certa criticidade na exigência do processo de fabricação do biscoito Cream Cracker em comparação aos demais produtos acabados, o qual necessita de diversas atividades operacionais para a sua finalização, resultando em um alto tempo total de produção.

Este trabalho objetivou analisar os métodos de produção executados pelos operadores e identificar problemas e soluções para a melhoria do processo de fabricação de biscoitos Cream Cracker com o auxilio do mapeamento de processos.

Além desta introdução, este trabalho foi organizado da seguinte maneira: a secção 2 traz alguns conceitos relevantes para contextualizar a pesquisa; a secção 3 descreve a metodologia usada; a secção 4 apresenta o estudo de caso; na secção 5 os resultados e análises são feitos; e, por fim, conclusão deste artigo são relatadas na secção 6.

\section{Fundamentação teórica}

\subsection{Estudo de métodos}

O método é um conjunto de atividades sistemáticas e racionais que, com maior segurança e economia, permite alcançar o objetivo - conhecimentos válidos e verdadeiros - traçando o caminho a ser seguido, detectando erros e auxiliando as decisões do cientista, segundo MARCONI e LAKATOS (2010, p.65).

MOREIRA (2008) observou que as técnicas e métodos associados à melhoria da produtividade servem tanto para auxiliar no diagnóstico de uma situação atual, como para acompanhar os efeitos de mudanças nas práticas de trabalho nas organizações. A partir disso, destaca-se que a principal técnica desenvolvida nesse âmbito é o estudo de 
tempos e movimentos, que tem um papel central na determinação da produtividade (PEINADO e GRAEML, 2007 apud VIEIRA et al., 2015).

O estudo de tempos e movimentos tem por objetivo determinar o tempo de cada atividade realizada no processo produtivo, auxiliando na proposição de possíveis melhorias para o ganho da produtividade organizacional, como são abordados na literatura. Além disso, auxilia na eliminação de trabalhos desnecessários e de gargalos da produção, enquanto contribui com métodos para mensuração do trabalho no intuito de determinar o melhor desempenho do operário (MORI et al., 2015).

Segundo os mesmos autores, para garantir a confiabilidade dos registros de tempo realizados para a determinação do tempo padrão do operador é preciso que se indique a quantidade de cronometragens essenciais para cada operação, de forma que o tempo real (TR), determinado pelo tempo de execução da atividade medido através do uso do cronômetro, seja ilustrativa.

Pela literatura básica, o número de ciclos cronometrados é determinado pela Equação (1) (MARTINS e LAUGENI 2005).

$$
N=\left(\frac{z \times R}{E r \times d 2 \times \bar{X}}\right)^{2}
$$

Em que:

- N: Número de ciclos cronometrados;

- z: Coeficiente de distribuição normal;

- R: Amplitude da amostra;

- Er: Erro relativo da medida;

- d2: Coeficiente em função do número de cronometragens realizadas preliminarmente;

- $\quad \bar{X}$ : Média dos valores das medições.

Os valores de $\mathrm{Z}$ e d2 utilizados no cálculo do número de ciclos são apresentados em tabelas encontradas na literatura (PEINADO e GRAEML 2007 apud FERREIRA et al., 2017).

O tempo normal é correspondente à duração de tempo necessária para a realização de uma atividade, sendo importante, neste caso, considerar a velocidade de desempenho do 
operador que realiza a mencionada tarefa. É importante frisar que, neste processo de determinação do tempo normal, o observador desempenha um papel fundamental, visto que, ele é o responsável por verificar as condições humanas do operador tendo como referência o ritmo normal por parte do operador nos outros dias de trabalho.

O observador pode levar em consideração, separadamente ou em conjunto, um ou mais fatores necessários para realizar o trabalho, como a velocidade de movimento, esforço, destreza, consistência, etc. (SLACK; CHAMBERS; JOHNSTON, 2015). Portanto, o tempo normal é o tempo real corrigido pela velocidade do operador em seu ritmo normal, conforme Equação (2).

$$
T N=T C \times V
$$

Em que:

- TN: tempo normal;

- TC: tempo cronometrado;

- $\quad V$ : velocidade.

O tempo de execução da atividade realizada sendo consideradas as tolerâncias do processo, é chamado de tempo padrão (MOKTADIR et al., 2017). Essas tolerâncias são relacionadas aos diversos fatores, como, tolerância pessoal, tolerância para a fadiga ou tolerância para espera, e são inevitáveis, interferindo diretamente na determinação do tempo das atividades. O fator de tolerância é calculado pelo tempo de tolerância que pode ser chamado também de tempo permissivo.

O tempo permissivo é definido pela razão entre o tempo não trabalhado pelo funcionário (tempo ocioso) pela carga horária de trabalho definido pela Equação (3) (MARTINS e LAUGENI, 2005). Com isso, o fator de tolerância pode ser obtido pela Equação (4).

$$
\begin{aligned}
& p=\frac{\sum \text { tempo de paradas }}{\text { Carga horária do turno de trabalho }} \\
& F T=\frac{1}{(1-p)}
\end{aligned}
$$

Em que: 
- $\quad p$ : tempo permissivo.

- FT: fator de tolerância;

Enquanto, o tempo padrão é obtido pelo tempo normal multiplicado pelo fator de tolerância seguindo a Equação (5).

$$
T P=T N . F T
$$

Em que:

- TP: tempo padrão;

- TN: tempo normal;

- FT: fator de tolerância.

\subsection{Gestão de processos}

Para o PNQ (Programa Nacional da Qualidade apud Souza, 2014), os processos são um conjunto de atividades inter-relacionadas que, conduzidas numa sequência lógica, produzem o resultado esperado e que atendem às expectativas e necessidades dos clientes. De forma resumida, segundo a NBR ISO 9000, os processos transformam insumos (entradas) em produtos ou serviços (saídas) (ABNT, 2000).

Dentre as abordagens da Gestão de Processos existe o ramo da Gestão de processos de negócios (ou Business Process Management - BPM, no inglês). Para HAVEY (2005), o BPM otimiza a automatização do fluxo de processos, aumentando a eficiência e a produtividade das atividades, além de garantir a formalização dos processos e dos pontos críticos de uma organização.

Ressaltam OLIVEIRA e NETO (2009) que a modelagem em BPM se faz primordial para um contexto operacional, pois viabiliza a consolidação dos processos. Após o mapeamento dos processos é possível entender com o levantamento do estado presente do negócio (as is), o melhor cenário ideal (should be) e a implementação mais indicada (to be), se assim o preferir.

Destacam-se algumas vantagens desta técnica de mapeamento: entendimento simples e objetivo; aplicação em processos simples e complexos; auxilia na localização de falhas; 
permite a melhoria contínua; e facilita a compreensão do processo (PAVANI e SCUCUGLIA, 2011).

\section{Metodologia}

O presente trabalho apresenta uma análise descritiva de dados relevantes que, quando interpretados por meio da aplicação de ferramentas qualitativas e quantitativas, possibilita o entendimento dos resultados e sugestões de melhorias com a finalidade de alcançar a descrição correta do processo produtivo e alertar sobre problemas ou potenciais aspectos negativos.

Desta forma, a Figura 1 representa as etapas essenciais da pesquisa realizada na empresa objeto de estudo, localizada em Pernambuco.

Figura 1: Etapas da pesquisa

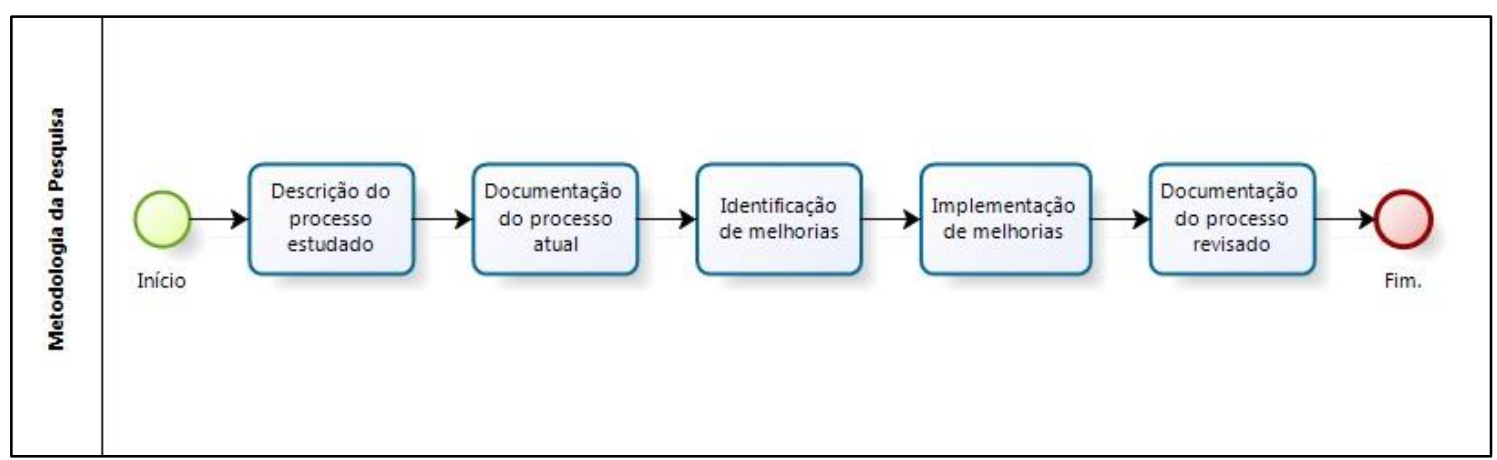

Fonte: Os autores (2019)

- Etapa 1 - Descrição do processo estudado: Nesta etapa foram identificados os objetivos, as saídas, os clientes, as entradas e componentes e os fornecedores do processo através da coleta de dados e informações da organização. Como também, a descrição do setor de preparação de massas e das atividades executadas neste processo.

- Etapa 2 - Documentação do processo atual: No intuito de tornar o processo analisado mais nítido e de fácil entendimento para a implementação de melhorias foi elaborado o mapeamento de processos com o uso do Software Bizagi Process Modeler, o qual é possível descrever o detalhamento das atividades realizadas por meio de representação gráfica. O mapeamento do processo foi elaborado de acordo com a coleta de dados e informações pelos supervisores e colaboradores da empresa do estudo de caso. 
- Etapa 3 - Identificação de melhorias: As melhorias foram identificadas por meio do estudo de tempos e movimentos, o qual foi descrito as atividades detalhadas do processo analisado e, consequentemente, a medição dos tempos de cada atividade da primeira fase de produção do biscoito Cream Cracker em uma amostra escolhida. Foram gravadas 5 medições da execução do trabalho operacional por meio de uma câmera de vídeo e utilizada uma folha de observações para anotações dos tempos mensurados durante visitas ao setor analisado.

- Etapa 4 - Implementação de melhorias: Para a implementação das melhorias foram feitos alguns ajustes nas atividades providenciando os materiais a serem utilizados na execução do novo método otimizado, juntamente com a realização do treinamento com os operadores, no intuito de padronizar as atividades. Após esses procedimentos, foram cronometrados os novos tempos das etapas executadas, utilizando os mesmos recursos citados anteriormente na etapa $3 \mathrm{com}$ a finalidade de obter resultados concretos.

- Etapa 5 - Documentação do processo revisado: Nesta parte foi feito um novo mapeamento do processo melhorado pelo uso do Software Bizagi Process Modeler (BPM) para a rápida visualização das reduções das atividades, e como forma de entendimento por parte dos gestores e colaboradores da organização.

\section{Estudo de caso}

A empresa escolhida para a realização deste estudo pertence ao segmento alimentício e está situada em Pernambuco, Brasil. Atualmente, a empresa possui duas fábricas, que operam em três turnos durante todos os dias do ano, exceto feriados, possuindo em média de 700 funcionários trabalhando a fim de atender a capacidade demandada do mercado. Portanto, é notável o crescimento das vendas durante esses últimos dois anos, pois a capacidade produtiva vem aumentando cada vez mais.

Ao observar todo o processo produtivo da linha 1 de biscoitos laminados na fábrica 2 foi possível analisar que o setor de preparação de massas é o que mais possui atividades mecânicas por parte dos operadores, composta em média por 5 operadores em cada turno. Cada um deles possui algumas atividades especificas no intuito de racionalizar o 
trabalho. Dentre todos os produtos produzidos pela unidade fabril, o biscoito Cream Cracker é o produto com o maior tempo de fabricação.

Basicamente, a preparação da massa é feita em dois processos:

- Preparação da esponja: possui este nome pela característica da massa finalizada nesta fase; as etapas do processo podem ser vistas na Figura 2:

Figura 2 - Processo de preparação da esponja do biscoito Cream Cracker

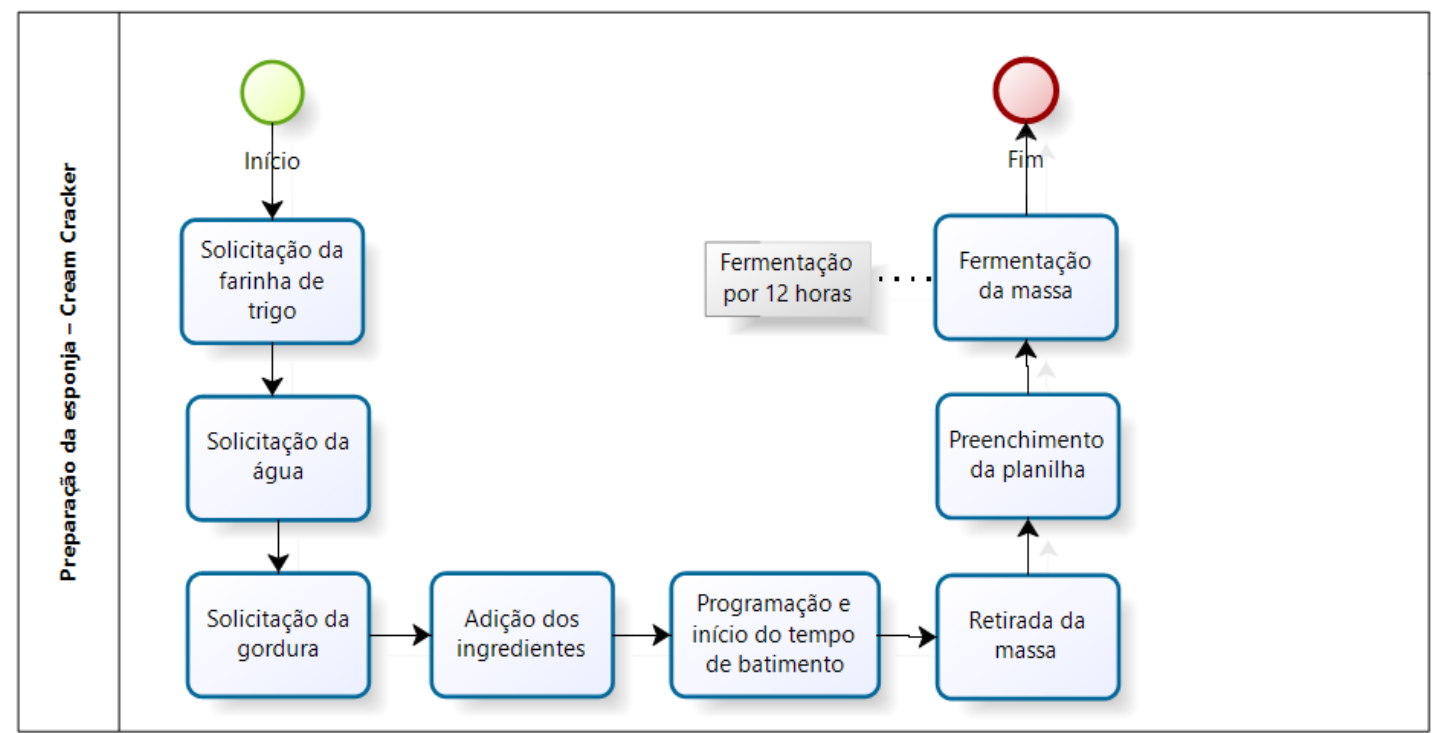

Fonte: Os autores (2019)

- Preparação do reforço: consiste na adição de ingredientes para obter a consistência, elasticidade e plasticidade da massa final; as etapas do processo podem ser vistas na Figura 3:

Figura 3 - Processo de preparação do reforço do biscoito Cream Cracker 


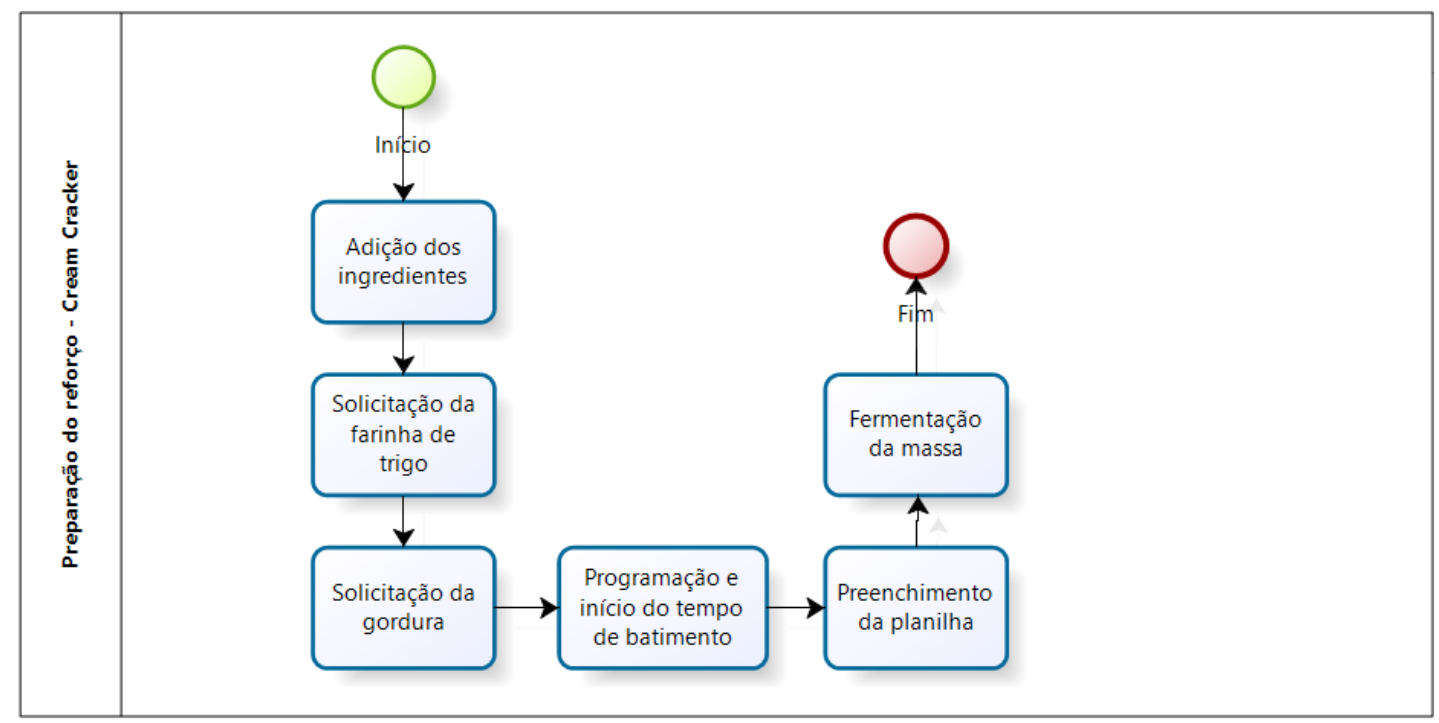

Fonte: Os autores (2019)

\section{Resultados e melhorias}

Com o intuito de aumentar a produtividade e eficiência do setor em questão foi realizado um estudo de tempos e movimentos das atividades desempenhadas pelos operadores da linha de biscoitos Cream Cracker. Foram feitas 5 cronometragens de cada etapa do processo de preparação de massas, especificamente, da primeira fase de produção (Esponja), que estão apresentadas na Figura 4.

Figura 4 - Tempo cronometrado para cada esponja preparada contida na amostra

\begin{tabular}{cccccccc}
\hline & Etapas da produção & $\mathbf{1}$ & $\mathbf{2}$ & $\mathbf{3}$ & $\mathbf{4}$ & $\mathbf{5}$ & $\begin{array}{c}\text { Cronometra } \\
\text { gem média } \\
\text { (min) }\end{array}$ \\
\hline $1 \quad$ Solicitação no Sistema de Dosagem & & & & & & $\mathbf{4 , 8 6}$ \\
& Automática & 4,80 & 4,82 & 4,86 & 4,90 & 4,92 & \\
2 & & & & & & $\mathbf{3 , 7 5}$ \\
3 & Adição dos ingredientes & 3,73 & 3,76 & 3,79 & 3,85 & 3,63 & $\mathbf{5 , 1 8}$ \\
4 & Batimento da massa & 5,18 & 5,18 & 5,18 & 5,18 & 5,18 & $\mathbf{3 , 7 1}$ \\
5 & Retirada da massa & 3,67 & 3,65 & 3,60 & 3,87 & 3,75 & $\mathbf{2 , 0 5}$ \\
\hline & Preenchimento da planilha & 2,05 & 2,00 & 2,03 & 2,07 & 2,1 & $\mathbf{1 9 , 5 5}$ \\
\hline
\end{tabular}

Fonte: Os autores (2019)

O tempo cronometrado (TC) ou tempo real (TR) foi obtido a partir das médias de cada etapa de produção da fabricação da esponja do biscoito Cream Cracker, evidenciando o maior tempo no batimento da massa e o menor tempo no preenchimento da planilha. Em relação ao tempo de batimento da massa ser constante é devido ao tempo automatizado e programado da batedeira. 
Para a escolha do número de ciclos necessários para o processo estudado em questão é preciso calcular o número de ciclos $(\mathrm{N})$ para cada etapa da produção em detrimento da Equação (1).

Como o processo possui 5 etapas de produção foi necessário calcular o número de ciclo para cada uma delas, utilizando o coeficiente de distribuição normal (z) com uma probabilidade de $90 \%$ que corresponde a 1,65 e um erro relativo de $10 \%$, sendo estes valores fixos para cada etapa. Além disso, foi preciso determinar o coeficiente em função do tamanho da amostra coletada (d2) que equivale a 2,326 correspondente a cinco amostras. Também, foi calculada a diferença do maior e menor valor do tempo total de preparar uma esponja de massa relacionada a amplitude (R). Como resultado obteve-se um número de ciclo aproximado de 01 para todas as etapas de produção. Portanto, o número de amostras coletadas anteriormente no total de 05 amostras possui a confiabilidade dos dados cronometrados.

O próximo passo é, então, a definição do tempo padrão (TP). Para isso, o fator de tolerância (FT) e o tempo normal $(\mathrm{TN})$ se fazem necessários previamente. Para o cálculo do tempo normal (TN), de acordo com a Equação (2), a velocidade analisada e considerada foi de $100 \%$ devido ao fato que o operador realizou as etapas de produção em seu ritmo normal de trabalho. Portanto, o tempo normal é igual ao tempo cronometrado $(\mathrm{TN}=\mathrm{TC})$.

Para o cálculo do fator de tolerância (FT) foi preciso utilizar os dados da carga horária de trabalho constituída por 8 horas diárias com 30 minutos de descanso para utilizar na Equação (3) e (4). De acordo com isso, o tempo permissivo (p) tabelado é de 0,0625 resultando em um valor de fator de tolerância (FT) de 1,067.

O tempo padrão foi calculado para cada atividade realizada do processo no intuito de determinar o tempo padrão total da fabricação de uma esponja de Cream Cracker pela Equação (5). O resultado se faz presente na Figura 5.

Figura 5 - Tempo padrão calculado para cada etapa de produção 


\begin{tabular}{lc}
\hline Etapas de produção & $\begin{array}{c}\text { Tempo } \\
\text { padrão (min) }\end{array}$ \\
\hline Solicitação no Sistema de Dosagem Automática & 5,18 \\
Adição dos ingredientes & 4,00 \\
Batimento da massa & 5,53 \\
Retirada da massa & 3,96 \\
Preenchimento da planilha & 2,19 \\
\hline Tempo padrão total $=$ & $\mathbf{2 0 , 8 6}$ \\
\hline
\end{tabular}

Fonte: Os autores (2019)

Com os dados somados de cada etapa da produção foi definido um valor de 20,86 minutos. Assim sendo, o tempo padrão para fabricação de uma esponja de massa de Cream Cracker aproximadamente é de 21 minutos.

\subsection{Proposta de melhoria}

Analisando todas as atividades executadas na primeira fase de fabricação do biscoito é possível identificar que existem algumas atividades manuais que podem ser otimizadas ao utilizar algumas ferramentas de trabalho ou na retirada de algumas atividades desnecessárias. Com isso, foram identificadas melhorias na etapa da solicitação no sistema de dosagem automática e na etapa de adição dos ingredientes.

A etapa da solicitação no sistema de dosagem automática é feita por parte manual na programação dos painéis e outra automática por parte da própria máquina. $\mathrm{Na}$ parte manual foi visto que o operador demorou mais que o normal devido a problemas mecânicos nos aparelhos, em especifico, na falha digital dos painéis. Portanto, a primeira proposta de melhoria seria ajustar as telas de configuração dos equipamentos.

Em relação à etapa de adição dos ingredientes, é uma atividade que exige a retirada dos lacres dos materiais a serem utilizados para despejar dentro da masseira, de forma que acaba aumentando o tempo de execução desta atividade. Com isso, as melhorias propostas seriam:

- O ajuste das telas de configuração dos equipamentos por parte da elétrica;

- A retirada dos lacres do fermento biológico e do açúcar cristal pesado antes do início da produção; 
- Utilização de uma ferramenta cortante para cortar facilmente o nó da embalagem da enzima protease enviada ao setor de preparação de massas pelo setor pré-mix;

- A futura aquisição de uma batedeira;

- A implantação de mais um painel do sistema de farinha de trigo.

De imediato foi sugerido trabalhar com as melhorias (1) e (2), pelo fato de serem fáceis de executar e de baixo custo.

Com as melhorias implementadas foram feitas as medições dos tempos de cada etapa do processo seguindo a Figura 6.

Figura 6 - Tempo cronometrado da melhoria para cada etapa do processo

\begin{tabular}{|c|c|c|c|c|c|c|c|}
\hline & Etapas da produção & 1 & 2 & 3 & 4 & 5 & $\begin{array}{c}\text { Cronometra } \\
\text { gem média } \\
(\mathrm{min})\end{array}$ \\
\hline 1 & $\begin{array}{c}\text { Solicitação no Sistema de Dosagem } \\
\text { Automática }\end{array}$ & 4,10 & 4,13 & 4,15 & 4,26 & 4,19 & 4,17 \\
\hline 2 & Adição dos ingredientes & 1,58 & 2,00 & 1,55 & 1,57 & 1,54 & 1,65 \\
\hline 3 & Batimento da massa & 5,18 & 5,18 & 5,18 & 5,18 & 5,18 & 5,18 \\
\hline 4 & Retirada da massa & 3,67 & 3,65 & 3,60 & 3,87 & 3,75 & 3,71 \\
\hline 5 & Preenchimento da planilha & 2,05 & 2,00 & 2,03 & 2,07 & 2,1 & 2,05 \\
\hline & Total & 16,58 & 16,96 & 16,51 & 16,95 & 16,76 & 16,76 \\
\hline
\end{tabular}

Fonte: Os autores (2019)

De acordo com esse tempo cronometrado foi feita a determinação do número de ciclos a serem cronometrados, o fator de tolerância (FT) e o tempo padrão (TP) utilizando os mesmos parâmetros da secção 5. Os números de ciclo de cada etapa da produção podem ser vistos na Figura 7.

Figura 7 - Número de ciclos cronometrados e calculado para cada etapa de produção da melhoria

\begin{tabular}{lcc}
\hline \multicolumn{1}{c}{ Etapas de Produção } & $\begin{array}{l}\text { Número de } \\
\text { ciclos }\end{array}$ & $\begin{array}{l}\text { Número } \\
\text { aproximado } \\
\text { de ciclos }\end{array}$ \\
\hline Solicitação no Sistema de Dosagem Automática & 0,07 & 1 \\
Adição dos ingredientes & 3,91 & 4 \\
Batimento da massa & 0 & 0 \\
Retirada da massa & 0,27 & 1 \\
Preenchimento da planilha & 0,12 & 1 \\
\hline
\end{tabular}

Fonte: Os autores (2019)

Como resultado obteve-se números de ciclo abaixo do número de amostras coletadas anteriormente (05 amostras), portanto, possui a confiabilidade dos dados cronometrados. 
Considerando uma eficiência de $100 \%$, o novo valor do tempo padrão (TP) pode ser visto na Figura 8.

Figura 8 - Tempo padrão calculado para cada etapa de produção depois da melhoria

\begin{tabular}{lc}
\hline Etapas de produção & $\begin{array}{c}\text { Tempo } \\
\text { padrão (min) }\end{array}$ \\
\hline Solicitação no Sistema de Dosagem Automática & 4,45 \\
Adição dos ingredientes & 1,76 \\
Batimento da massa & 5,53 \\
Retirada da massa & 3,96 \\
Preenchimento da planilha & 2,19 \\
\hline Tempo padrão total $=$ & $\mathbf{1 7 , 8 9}$
\end{tabular}

Fonte: Os autores (2019)

É possível notar uma redução do tempo padrão para fabricação de uma esponja de massa de Cream Cracker antes e depois da melhoria implementada de 2,97min evidenciando a redução dos desperdícios de tempos e movimentos.

Após a escolha de uma das etapas a ser melhorada foi elaborado o mapeamento das atividades pelo Software Bizagi Process Modeler para demonstrar o novo rearranjo das atividades. Na configuração inicial pode-se observar que as atividades seguem um fluxo linear de produção, como mostra a Figura 9.

Figura 9 - Subprocesso da adição dos ingredientes antes da melhoria proposta

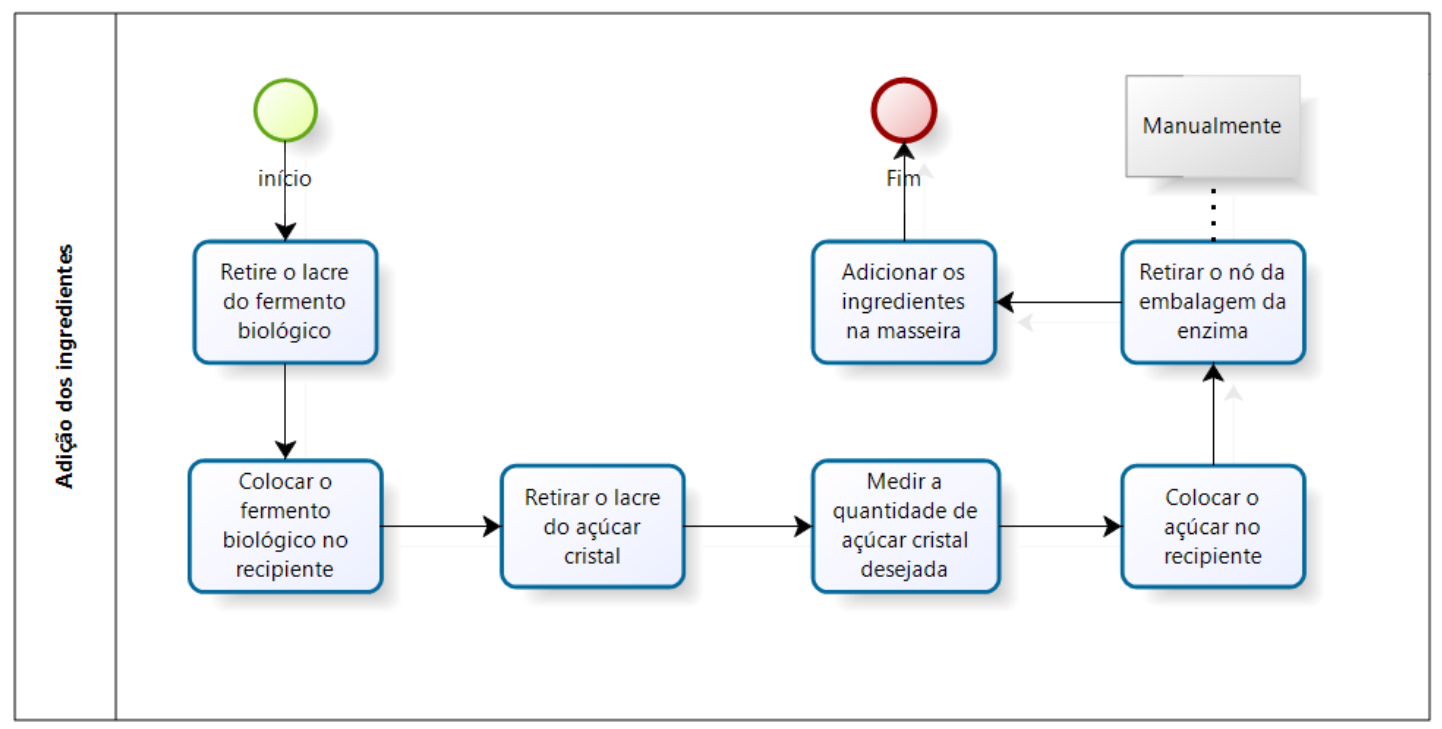

Fonte: Os autores (2019) 
Após o rearranjo das atividades, parte das atividades é feita paralelamente e com o auxílio de uma ferramenta cortante para facilitar a retirada do nó na embalagem da enzima, como pode ser visto na Figura 10.

Figura 10 - Subprocesso da adição dos ingredientes depois da melhoria proposta

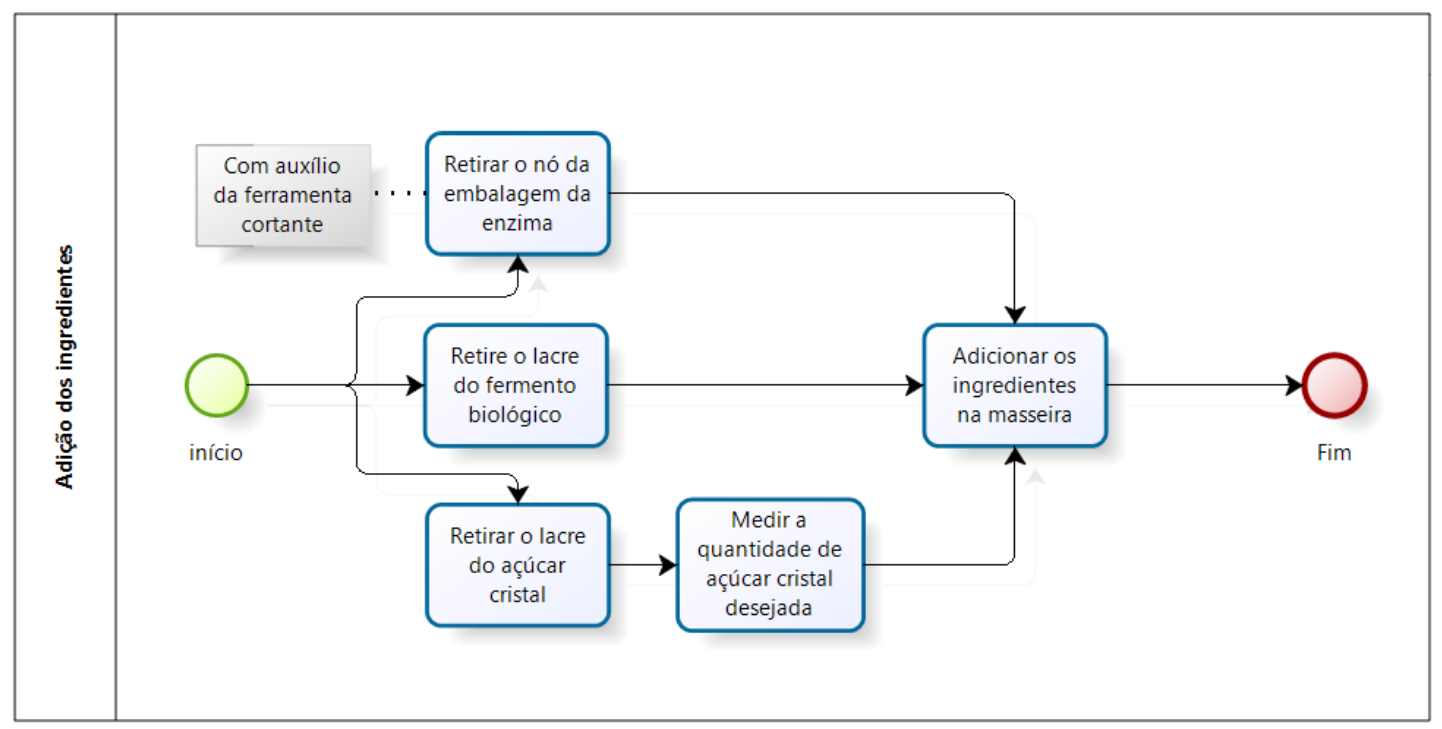

Fonte: Os autores (2019)

Com a distribuição das atividades na Figura 11 foi possível verificar a redução de duas atividades no subprocesso estudado ocasionando em uma redução do tempo total deste subprocesso. Vale ressaltar que as atividades em paralelo consomem menos tempo que as atividades em linha contínua, ou seja, as atividades podem ser executadas ao mesmo tempo por operadores diferentes resultando assim na eficiência da produtividade.

\section{Conclusão}

As empresas passam, cada vez mais, por uma série de transformações dentro dos processos de tomada de decisões de negócios para garantir o nível de excelência para os seus clientes necessitando reduzir os tempos de fabricação dos produtos oferecidos ao mercado, além de diminuir os custos de fabricação e de aumentar a produtividade e eficiência dos processos, atingindo o aumento da lucratividade.

Sendo assim, esta pesquisa buscou estudar e entender o processo do setor de fabricação do biscoito Cream Cracker, produto escolhido por sua criticidade de produção, fabricado em uma indústria alimentícia da região do Agreste de Pernambuco. A partir deste estudo, foi possível identificar os fatores que influenciam na queda da 
produtividade, por meio do estudo de tempos e movimentos com o auxílio do mapeamento de processos para a implementação de melhorias.

Com todo o estudo realizado foi possível observar que o uso da ferramenta Bizagi Process Modeler auxiliou na representação gráfica das atividades do processo, possibilitou também o entendimento com nitidez do processo analisado, facilitando assim a compreensão das atividades que foram reajustadas e outras que foram eliminadas para a diminuição dos tempos de produção evidenciados pelos resultados obtidos da pesquisa, conseguindo alcançar os objetivos pretendidos.

Desta forma, a pesquisa contribui para a disseminação do conhecimento sobre estudo de tempos e movimentos em relação ao setor da indústria alimentícia. Assim, é primordial afirmar o quão é relevante o poder da tomada de decisão correta levando em consideração as limitações da organização para o sucesso da implementação de melhorias no processo, promovendo assim a satisfação da empresa e dos seus consumidores.

\section{REFERÊNCIAS}

ABNT, Associação Brasileira de Normas Técnicas. NBR ISO 9001:2008, Sistemas de Gestão da Qualidade - Requisitos. Rio de Janeiro: ABNT, 2008.

FERREIRA, Letícia Ali Figueiredo; DOS SANTOS, Ana Carla de Souza Gomes.; DIAS, Josinaldo de Oliveira; PESSANHA, Luiz Phillipe Mota. Engenharia de Métodos: uma Revisão de Literatura sobre o Estudo de Tempos e Movimentos. Revista FATEC Zona Sul v.4, n.3, edição especial, Abril/2018.

HAVEY, Michael. Essential Business Process Modeling. O’Reilly, August 2005.

MARCONI, M. de A.; LAKATOS, E. M. Fundamentos da metodologia científica. 7. ed. São Paulo: Atlas, 2010.

MARTINS, Petrônio G.; LAUGENI, Fernando P. Administração da produção. 2.ed. rev., aum. e atual. São Paulo: Saraiva, 2005.

MOREIRA, Daniel Augusto. Administração da produção e operações. 2. ed. rev. amp. São Paulo: Cengage Learning, 2008.

MOKTADIR, Md. Abdul; AHMED, Sobur; TUJ-ZOHRA, Fatema; SULTANA, Razia. Productivity Improvement by Work Study Technique : A Case on Leather Products Industry of Bangladesh. Industrial Engineering Management, v. 6, n. 6, p. 1-11, 2017.

MORI, Vipulkumar V.; KANCHAVA, Yuvrajsinh B.; KARETHA, Pravin A.; CHAROLA, Manish B. Productivity Improvement by use of Time Study, Motion Study, Lean Tool's and Different Strategy for Assembly of Automobile Vehicles. International Journal for Scientific Research \& Development, v. 3, n. 2, p. 2321-613, 2015 . 
OLIVEIRA, Saulo Barbará de; NETO, Mario de Araújo Almeida. Análise e modelagem de processos. In: VALLE, Rogério; OLIVEIRA, Saulo Barbará de. Análise e modelagem de processos de negócio. São Paulo: Atlas, 2013. p.37-51.

PAVANI, Orlando Júnior; SCUCUGLIA, Rafael. (2011), Mapeamento e gestão por processos - BPM.

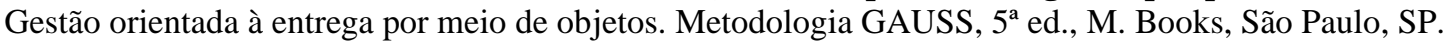

SLACK, Nigel; CHAMBERS, Stuart; JOHNSTON, Robert. Administração da produção. 4.ed. São Paulo, Atlas, 2015.

VIEIRA, Romero Rondinele Dos Santos; CORREIA, Ana Maria Magalhães; LUCENA, André Duarte; SILVA, Armistrong Martins. Estudo de Tempos e Métodos no Processo Produtivo de uma Panificadora Localizada em Mossoró/RN. Revista Eletrônica Gestão \& Sociedade, v.9, n.23, p. 977999, Maio/Agosto - 2015. 